\title{
ZENER-LIKE BREAKDOWN IN DIELECTRICS AT OPTICAL FREQUENCIES
}

\author{
V. L. Komolov \\ St. Petersburg National Research University of Information Technologies, Mechanics and Optics, Kronverkskiy 49, \\ 197101 St. Petersburg, Russia \\ E-mail: vlkomolov@gmail.com
}

Received 18 November 2013; accepted 4 December 2013

\begin{abstract}
A possible mechanism of arising of strong induced absorption in transparent dielectrics under the action of a high-power short light pulse is discussed. A simple qualitative analysis has been carried out under the assumption that the absorption in the transparent medium arises due to the excitation of electrons to the conduction band by the resonant tunnel transitions between the real energy levels arising in the system under the intensive light action.
\end{abstract}

Keywords: tunnelling, light action, optical breakdown, resonant transitions

PACS: 81.07.-b, 81.16.-c

\section{Introduction}

The problem of adequate description of the intrinsic optical breakdown in a transparent material under the action of a short pulse of intense optical radiation arose in the early 80 s of the last century [1, 2].

Despite many attempts to create a model of this phenomenon, today we have no proper description of the available set of experimental data. The main reason for this is the absence of a satisfactory model of induced absorption arising in the transparent medium during the light action.

It should be recalled that the intrinsic optical breakdown of a transparent medium is characterized by the following features formulated in 1985 [3] and confirmed later in a wider range of irradiation conditions [4]:

(1) There is no pre-threshold matrix ionization.

(2) The breakdown occurs at the maximum power density and progresses in a very short time (less than ten ps).

(3) By reducing the peak intensity to a few percent lower than the threshold, no damage occurs even though the effective duration of the light action increases by at least an order of magnitude.

(4) The threshold power density $q^{*}$ is determined by the properties of dielectrics and keeps its value with an accuracy determined by the measurement precision $( \pm 2 \%)$ in a wide range of pulse durations.

(5) For K8 glass, this value is $\sim(6-9) \cdot 10^{12} \mathrm{~W} / \mathrm{cm}^{2}$ $\left(\sim(6-8) \cdot 10^{7} \mathrm{~V} / \mathrm{cm}\right)[3$, 迎.
Among the main processes that can be responsible for the nonlinear photo-excitation of the system and give rise to significant concentrations of free carriers in the medium and, finally, to its optical breakdown, traditionally considered are the processes of avalanche ionization and multi-photon absorption [1, 2]. Unfortunately, the validity of the proposed models is rather doubtful.

None of the traditional models describes the extremely sharp behaviour of the breakdown threshold when the reduction in the intensity of light by only a few percent does not lead to a decrease in light transmission even with the growth of pulse width to five orders of magnitude (at the time range of $10^{-8}-10^{-13} \mathrm{sec}$ ). At the same time, at the threshold intensity one can observe almost the complete "cut-off" of the transmitted light - the "metallization" of dielectrics. The possibility of such metallization was pointed in 1986 in [5]; however, no specific mechanism of this process has been proposed.

In the present work, we propose an approach to the description of photo-excitation of the system, taking into account experimentally observed features of a breakdown mentioned above.

Process analysis is carried out using the concepts of electron tunnelling into the conduction band. This choice is due to the fact that the tunnelling effect occurs instantly, as the tunnelling characterizes not a transition from one state to another, but the probability of finding an electron in a given state. 
The electron responds instantly to changes in the total potential of the system.

The boundary between the modes of multi-photon and tunnelling ionization is determined by the so-called adiabatic parameter

$$
\gamma=\frac{\hbar \omega}{\Delta}
$$

where $\omega$ is the frequency of the light wave, $\Delta$ is the characteristic width of the gap in the energy spectrum of the system (ionization potential, band gap, the spacing between energy levels of the system, etc.).

If $\Delta$ is the band gap $E_{\mathrm{g}}$ of the material, we have for a selected set of parameters $\left(\Delta=E_{\mathrm{g}}=7 \mathrm{eV}, \lambda=1.06 \mu \mathrm{m}\right)$ typical of the optical breakdown studies the value of the adiabatic parameter of about $\gamma \approx 0.17<<1$. Then the analysis of photo-excitation of the medium can be carried out in terms of tunnelling in the adiabatic limit, i. e. under assumption that the probability of tunnelling of electrons into the conduction band tracks an instantaneous value of the amplitude of light wave field.

\section{Model and results}

\subsection{Zener tunnelling}

Let us estimate the possible role of tunnelling in the photo-excitation of dielectrics. For this purpose we will extend the Zener approach [6], which has been proposed to describe the breakdown in a constant electric field for a variable high-frequency field; this seems to be reasonable as long as the adiabatic parameter $\gamma<<1$.

To estimate the probability $P$ of electron location in the conduction band we use the standard theory of Zener [6], which gives the following expression for $P$ :

$$
P=\exp \left(-\frac{\pi^{2} m a}{h^{2}} \cdot \frac{E_{\mathrm{g}}^{2}}{e F}\right),
$$

where $e$ and $m$ is the charge and mass of the electron, respectively, $E_{\mathrm{g}}$ is the band gap of the material, $F$ is the instant value of the electric field in the light wave, $a$ is the lattice parameter, and $h=2 \pi \hbar$ is the Planck constant.

The concentration of electrons that penetrate into the conduction band during one half-cycle of the light wave oscillation is equal to

$$
n_{1}=N \cdot P=\frac{1}{a^{3}} \cdot \exp \left(-\frac{\pi^{2} m a}{h^{2}} \cdot \frac{E_{\mathrm{g}}^{2}}{e F}\right),
$$

where $N=1 / a^{3}$ is electron density in the valence band.

We must keep in mind that as we speak about the tunnelling process, the transition occurs instantly and does not require any energy. The probability $P$ is sim- ply the probability of the location of an electron in the conduction band.

When the electron oscillates in the presence of an external electric field $F$ with a frequency (Bloch oscillations), an equilibrium between the valence and conduction bands arises, and the density of conduction electrons after the first oscillation reaches the level of $n_{1}=N \cdot P$ [7]. At the end of the half-cycle of the light wave just this concentration of electrons remains in the conduction band. With each next halfwave, the process is repeated and the total concentration is determined by its accumulation in the upper band during the pulse $\tau_{\mathrm{p}}$.

Figure 1 shows calculation results of the concentration of electrons $n_{1}$, which excited into the conduction band for one half-wave on the light intensity. The calculation has been carried out for the following set of parameters: ( $a=3.5 \AA, E_{\mathrm{g}}=7 \mathrm{eV}, e$ and $m$ charge and mass of free electron, respectively). It can be seen that when the light intensity is close to the optical breakdown threshold, about $10^{20} \mathrm{~cm}^{-3}$ free carriers instantly (at $10^{-15} \mathrm{sec}$ ) arise in the conduction band, and they can cause subsequently the usual thermal damage of the transparent material due to absorption of the following part of the light pulse.

However, the validity of this approach is questionable. The above estimates predict a significant concentration of electrons in the conduction band at light intensities below the breakdown threshold. This conclusion is not consistent with experimental data [3, 4. which claim that at the intensity that is only a few percent below the threshold no changes in the light absorption have been detected. But noticeable light absorption surely should arise at such high density of free carriers. The same can be said about the models of avalanche and multi-photon ionization. In both cases

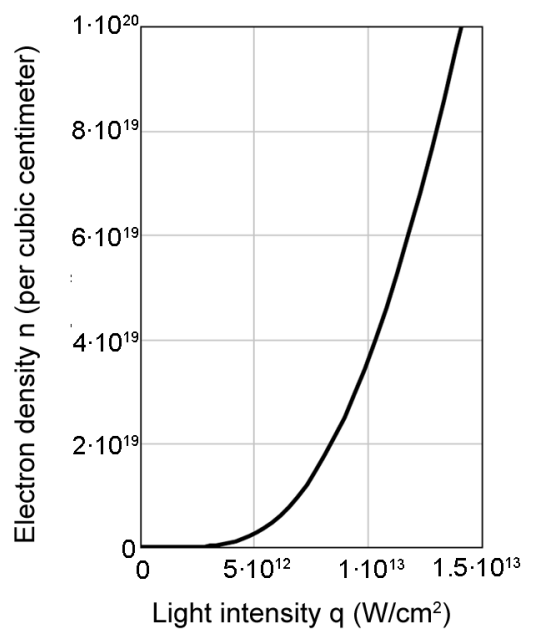

Fig. 1. Dependence of electron density $n_{1}$ in the conduction band on light intensity $q$. 
the growth rates of electron density on light intensity are not high enough to explain experimental data.

\subsection{Possible resonances and the shifts of energy levels}

The above estimates do not take into account an important fact that strongly affects the process of medium photo-excitation, namely, the changing of the structure of energy levels under the action of an external field, for example, the shift of the states due to the Stark effect. As the probability of the existence of an electron in a particular state noninertially tracks the instantaneous value of full potential, the strong changes of ionization rate can be expected.

It will be shown below that an accounting of displacement of the energy levels of the system in an external electric field can lead to the appearance of resonances in the system due to the equality of the frequencies of Bloch oscillations of the valence electron and the light wave and even to a collapse of the forbidden band.

In previous estimations we did not take into account two important factors that can affect strongly the process of photo-excitation protection:

1. The possible occurrence of resonances in the system when the frequencies of Bloch oscillations and the light wave become equal.

2. Shifts of energy levels in the electric field due to the Stark effect.

It can be expected that both of these factors can lead to an almost instantaneous "metallization" of dielectrics. In this section we shall try to estimate the possible influence of the above factors on the process of photo-excitation of dielectrics.

The possibility of resonance occurrence under the action of optical radiation on dielectrics can be demonstrated in terms of classical physics. It is known that under the action of the external field with instant strength $F$ the electron oscillates with the Bloch frequency $\omega_{\mathrm{el}}=\frac{e \cdot F \cdot a}{\hbar}$, 8 .

If the frequency of the external light field $\omega$ becomes close to $\omega_{\mathrm{el}}$, the resonance occurs in the system and the amplitude of the electron oscillations $Z$ increases dramatically, far exceeding the size of a crystal cell $a$ :

$$
Z \approx \frac{e \cdot F}{m\left(\omega^{2}-\omega_{\mathrm{el}}^{2}\right)} .
$$

In other words, the electron is no longer tied to its "own" cell and becomes delocalized - "metallization" of the medium takes place.

Figure 2 shows that for our case $(a=3.5 \AA$, $\left.\omega=1.78 \cdot 310^{15} \mathrm{sec}^{-1}\right)$ resonance occurs at light intensities $q \sim 10^{12} \mathrm{~W} / \mathrm{cm}^{2}$, slightly less than the measured breakdown threshold [3, 他.
In terms of quantum mechanics, the possibility of resonance can be described as follows. Under the action of the external field with instant strength $F$, the electron oscillates with the Bloch frequency $\omega_{\mathrm{el}}=(e \cdot F \cdot a) / \hbar$.

Its behaviour is qualitatively similar to the behaviour of a harmonious oscillator, in particular, the energy eigenvalues form a system of equidistant levels, the so-called Wannier-Stark ladder, $E_{\mathrm{k}}= \pm k \cdot \Delta E$ $(k=0, \pm 1, \pm 2, \ldots)$, the spacing between which $\Delta E=e \cdot a \cdot F$ is determined by the instant value of the external field. At moderate fields $\left(\sim 10^{5}-10^{6} \mathrm{~V} / \mathrm{cm}\right)$ this spacing is small compared to the characteristic energy values, but in our case, when the light intensity $q$ reaches the value of $\sim 10^{13} \mathrm{~W} / \mathrm{cm}^{2}$ and the corresponding field strengths $F \sim 10^{7}-10^{8} \mathrm{~V} / \mathrm{cm}$ are comparable to intraatomic, shifts of the levels become large and should be taken into account in analysis of excitations in solids. In other words, the energy $\Delta E=\hbar \omega_{\mathrm{el}}=e \cdot F \cdot a$, the typical value of the splitting and shifting of the energy levels under the action of an external field, is just the criterial parameter of the problem.

Figure 3 shows a plot of the level shift $\Delta E=e F a$ for the different values of the light intensity. The straight line indicates the value of the photon energy $\hbar \omega$, and the dash-dot line indicates the band gap $E_{\mathrm{g}}$. It should be noted that the level's shift tracks the instantaneous value of the field strength. To simplify the estimations it was assumed that the field strength does not vary in a sinusoidal manner, but consists of a set of rectangular "half-waves" of the same frequency as the real light wave does but with constant field strength value $F$ at each half-cycle.

It can be seen from the curves of Fig. 3 that even at the light intensity $q \approx 10^{12} \mathrm{~W} / \mathrm{cm}^{2}$ the value of $\Delta E$

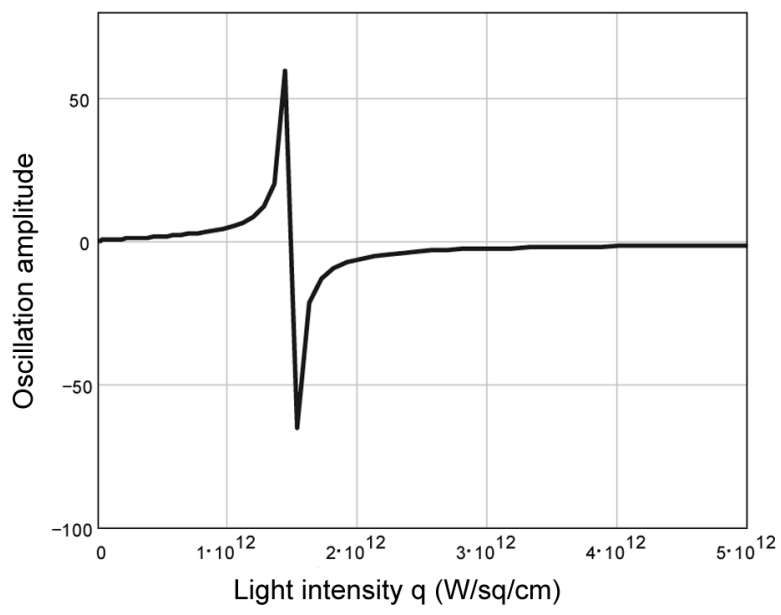

Fig. 2. Amplitude of electron oscillations under the action of the light with frequency $\omega$. The resonance is achieved at light intensities close to the experimental value of the threshold breakdown. 


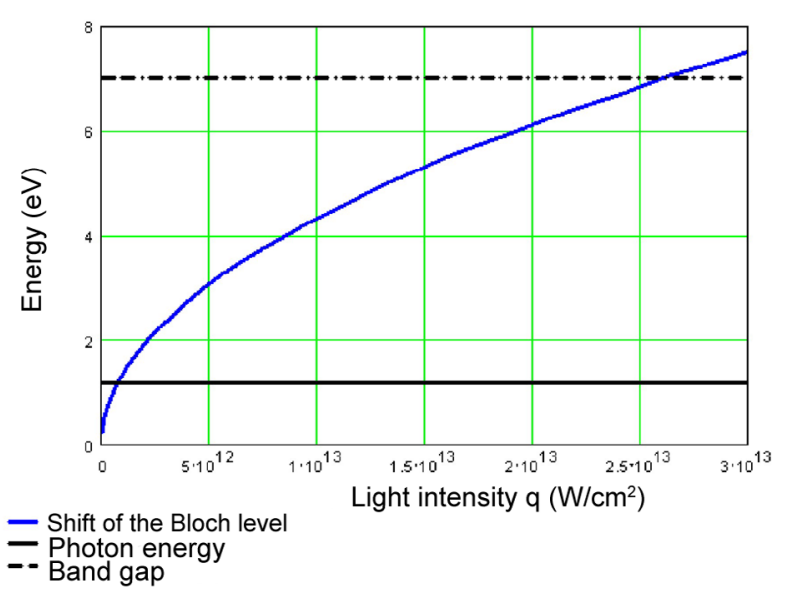

Fig. 3. Dependence of energy level shift on light intensity.

becomes comparable to the photon energy. Since under the field action a system of levels with energy $E_{\mathrm{k}}= \pm k \cdot \Delta E$ arises in the solid, it is natural to assume that the excitation of electrons to the conduction band is the result of successive resonant tunnel transitions between the suitable "rungs" of the ladders at the valence and conduction bands. The probability of such transitions seems to be sufficiently high for practically instantaneous "metallization" of dielectric medium, since they correspond to resonant transitions between the real levels.

It should be noted that the curves in Fig. 3 show also that at intensities of about $6 \cdot 10^{12} \mathrm{~W} / \mathrm{cm}^{2}$ the shift of energy levels reaches the value equal to the half of the band gap, so the complete crossing of the levels from different bands and collapse of the band gap become possible.

\section{Discussion and conclusion}

First of all, it should be emphasized that the only goal of this paper is to find a possible way for correct description of the initial stage of the optical breakdown.

The above estimations are too rough to make an exact conclusion about the mechanism of induced absorption in the medium, but we hope that they properly describe the qualitative picture of the processes occurring in the medium. It should be noted that a peculiarity of the proposed model is that it is self-adjusting.
Under the influence of the field, all modifications of level's structure occur instantly, and the spacing between them is changing, tracking the instant value of the external field. And when the shift of the levels reaches the desired value, a tunnel transitions through the reduced forbidden band or even the collapse of band gap become possible.

Comparing the proposed model predictions with the experimental data listed at the beginning of this paper, it should be noted that it can explain, at least qualitatively, some experimental facts, namely:

1. The absence of sub-threshold matrix ionization, since slight deviation from resonance reduces the ionization rate of the solid extremely fast.

2. The resonant conditions are determined only by the instant value of the field (light intensity), the resonant transitions occur without any delay.

3. Even very rough estimates of the threshold values of the light intensity given above are in reasonable agreement with the available experimental data.

So, it can be expected that the proposed approach to the description of the initial stage of optical breakdown can be promising for the creation of a correct model of the optical breakdown in dielectrics.

\section{References}

[1] A.A. Manenkov and A.M. Prokhorov, Phys.Usp. 29, 104-122 (1986).

[2] V.P. Veiko, M.N. Libenson, G.G. Chervyakov, and E.B. Yakovlev, Interaction of the Laser Radiation with the Matter (Moscow, 2008).

[3] O.M. Efimov, The Nonlinear Absorption of Laser Radiation and Optical Breakdown of Silicate Glasses, $\mathrm{PhD}$ thesis (Leningrad, 1985).

[4] O.M. Efimov, J. Opt. Technol. 71(6), 338-347 (2004).

[5] L.B. Glebov, O.M. Efimov, M.N. Libenson, and G.T. Petrovskii, Sov. Phys. Dokl. 31, 326 (1986).

[6] C. Zener, Proc. Roy. Soc. London A 145, 523-529 (1934).

[7] A.S. Zingerman, Usp. Fiz. Nauk 46, 450-507 (1952).

[8] B.K. Ridley, Quantum Processes in Semiconductors (Oxford, 1999).

\section{ZINERIO TIPO PRAMUŠIMAS DIELEKTRIKUOSE ESANT OPTINIAMS DAŽNIAMS}

\section{L. Komolov}

